\title{
473737 - GENERAL ANESTHESIA WITH OR WITHOUT THORACIC EPIDURAL BLOCK FOR CARDIAC SURGERY - A DECISION ANALYSIS
}

\author{
Anthony Ho, MD, FRCPC, FCCP ${ }^{1}$, Anna Lee, PhD $^{1}$, Gavin Joynt, MB, FCCP ${ }^{1}$, \\ Manoj Karmakar, MD, FRCA ${ }^{1}$, Paul Myles, MD, FANZCA ${ }^{2}$ \\ 1. Anaesthesia and Intensive Care, The Chinese University of Hong Kong, Hong \\ Kong SAR, China
}

2. Anaesthesia and Perioperative Medicine, Monash University, Melbourne, VIC, Australia

Introduction: Despite a growing interest in the use of thoracic epidural anesthesia/analgesia (TEA) to supplement general anesthesia (GA) in adult cardiac surgery, the issue remains controversial. We decision analyzed from the patient's perspective two management options for adult cardiac surgery: GA vs. GA + TEA, and determined the probability weighted utilities of both options and the influence of changing parameter values on analysis outcome.

Methods: We constructed a probabilistic decision tree that included the possibility of epidural hematoma (2) or abscess, incomplete and complete paraplegia, myocardial ischemia/infarction (MI) (2), pulmonary and renal impairment, miscellaneous morbidity, and death. All relevant data (probabilities and utility values) were, where possible, derived from the literature. Where uncertainties existed, the decision analysis results were sensitivity analyzed to determine the influence of parameter changes on the final decision.

Results: Patients undergoing cardiac surgery in the absence of coronary artery disease would not derive a net benefit from TEA. For coronary artery bypass grafting, old patients may derive a net benefit from TEA but the precise cutoff age, somewhere between 70-80 years, is difficult to determine. The reasons for not using TEA become increasingly compelling as the age of the patient decreases. The decision would tip in favor of TEA, however, should it ever be shown that TEA significantly reduces MI, which so far, it has not.

Discussion: Given the lack of strong evidence that TEA reduces MI, TEA should only be used, if at all, on the very elderly coronary artery bypass patient. Proponents of the technique should focus on exploiting its potential cardiac protective properties because of the great influence of MI on morbidity and mortality, and the ultimate decision on the utility of TEA.

References: Ho AMH, et al. Chest 2000; 117:551-5; Liu SS, et al. Anesthesiology 2004;101:153-61

Threshold analysis of parameters 


\begin{tabular}{|c|c|c|}
\hline Parameters & Favors block & Favors no block \\
\hline Patient age (years) & $\geq 77$ & $<77$ \\
\hline Risk of epidural hematoma & $<1 / 3700$ & $>1 / 3700$ \\
\hline Paraplegia Quality of Life reduction factor & $<0.66$ & $>0.66$ \\
\hline Postop Quality of Recovery reduction (block) & $>0.168$ & $<0.168$ \\
\hline Postop Quality of Recovery reduction (no block) & $>0.58$ & $<0.58$ \\
\hline Risk of epidural abscess & $<1 / 50000$ & $>1 / 50000$ \\
\hline
\end{tabular}

